\title{
诱导产生多能性干细胞(iPS 细胞)的研究进展
}

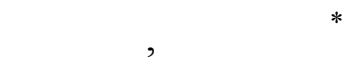

(1) 中国科学院动物研究所计划生育生殖生物学国家重点实验室, 北京 100101;

(2) 中国科学院研究生院, 北京 100049

*联系人, E-mail: duane@ioz.ac.cn

2007-12-11 收稿, 2008-01-11 接受

国家重点基础研究发展计划(批准号: 2007CB947401)和国家载人航天工程(批准号: 921-2)资助项目

摘要 最近, 将人类体细胞诱导成为多能性干细胞(induced pluripotent stem cells, iPS 细胞)的研究成果在生命科学领域引起了又一次轰动, 被誉为人类生命科学新的里程 碑. 这些突破性进展为进一步研究多能性的调控机制带来了观念上的创新并提供了 新的研究模型, 同时也建立了一种全新的体细胞核重编程的方法. 由于这种方法相对 容易操作, 而且比较稳定, 因而在生物学基础研究和临床应用方面具有潜在的价值. 本文从 iPS 细胞的研究历程、获得 iPS 细胞的几个关键步骤、 iPS 细胞研究所引发的 相关科学问题以及 iPS 细胞的应用价值和发展方向 4 个方面进行了评述.

关键词

诱导产生的多能性干细胞 重编程

多能性

胚胎干细胞

分化
2006 年, Takahashi和Yamanaka ${ }^{[1]}$ 将几个转录因 子导入已分化的小鼠皮肤成纤维细胞, 进而获得了 类似于胚胎干细胞(embryonic stem cells, ES细胞)的 多能性干细胞, 称之为 “诱导产生的多能性干细 胞”(induced pluripotent stem cells, iPS细胞). 这一研 究明确地证实了分化的细胞可以通过少数几个因子 的外源导入而被重编程到具有多能性的状态, 因而 受到了整个生命科学领域的广泛关注. 本文将对这 一新进展进行概述, 其中着重描述一些关键环节, 并 探讨相关的科学问题.

\section{1 细胞重编程的研究概况}

细胞的多能性 (pluripotency) 是指其能够分化为 机体内各种细胞类型的潜能 ${ }^{[2]}$. 细胞核重编程是指, 成熟的细胞由分化的状态被逆转到一种未分化状态 的过程 ${ }^{[3]}$. 有研究显示小鼠胚胎早在 2-细胞期到 4-细 胞期的时候, 各个卵裂球很可能就已经体现出了不 同的发育倾向 ${ }^{[4]}$. 此后, 细胞即向着不同的方向不断 地发生分化. 无论细胞分化的程度如何, 它们与未分 化细胞在基因组成上都是一样的. 然而, 在其发育过 程中, 它们的基因组发生了许多表观遗传修饰, 基因
的表达状况发生了改变, 由此产生了各异的分化表 型. 表观遗传修饰是可逆的, 因此, 成熟的细胞也能 够被逆转到未分化的状态. 目前, 已经有几种方法可 以实现体细胞的重编程.

首先, 细胞核的重编程现象最早是在核移植实 验中被发现的 ${ }^{[5]}$. 核移植实验最初是在两栖动物中实 现的, 通过将体细胞核注入到去核卵母细胞中. 迄今, 人们已经获得了多种核移植动物, 也建立了来源于 核移植胚胎的胚胎干细胞系. 核移植实验明确证明 了分化的体细胞可以被逆转到全能的状态, 并且可 以用于区分遗传学和表观遗传学的变化, 意义十分 重大. 然而, 体细胞核移植实验也具有很大的局限性: 克隆的效率极低; 产生的许多后代在各个阶段都体 现出严重的发育异常; 由于需要使用卵母细胞, 核移 植实验在人类中的应用受到强烈的伦理学质疑. 这 些不足, 都制约了核移植技术的进一步发展和应用.

其次, 将成熟的体细胞与多潜能的细胞 (ES细 胞、胚胎癌细胞(EC细胞)等)融合 ${ }^{[6 \sim 9]}$, 或者用胚胎干 细胞提取物来处理体细胞 ${ }^{[10,11]}$, 也可以在一定程度 上使体细胞发生重编程. 利用这两种方法, 可以实现 某些多能性标志分子的重新表达和多种分化潜能的 
获得. 这两种方法为研究重编程的基因调控以及分 析重编程的生物化学和动力学变化提供了可能性. 但是这两种方法也有明显的弊端. 体细胞与多能性 细胞的融合效率比较低, 融合之后的细胞具有两套 染色体, 并且在移植后会发生排斥现象, 这就制约了 细胞融合的临床应用. 而用多能性细胞的提取物处 理体细胞的方法在目前还存在一定争议, 这种方法 所诱导的重编程效果也十分有限.

此外, 多能性干细胞可以由某些类型的细胞在 体外培养时自发产生. 内细胞团在体外培养产生多 能性的胚胎干细胞; 原始生殖细胞(PGCs) 在培养时 能形成多能性的胚胎生殖细胞 $(\mathrm{EG} \text { 细胞 })^{[12]}$; 成年动 物骨髓来源的细胞能在长期体外培养后形成多能性 成体前体细胞(MAPCs) ${ }^{[13]}$; 精原干细胞在体外培养 时偶尔能形成类似ES细胞的多能性干细胞 ${ }^{[14,15]}$.

上述方法大多需要多能性细胞来提供重编程所 需的复杂条件, 重编程的效率和程度也非常有限, 这 些方法的广泛应用受到各种因素的限制。通过外源 导入与多能性相关的转录因子来诱导体细胞核发生 重编程, 从而使体细胞转变成多能性干细胞, 是一种 具有很强创新性的研究方法, 这种新方法与上述几 种方法相比, 相对简单, 摆脱了材料来源和伦理学的 诸多限制, 重编程的效率和程度都十分可观, 因而该 研究成果引起了生命科学领域一次巨大的轰动, 对 今后重编程研究的发展也将起到强有力的推动作用.

\section{2 iPS 细胞的研究概况}

iPS细胞首先是由Takahashi和Yamanaka在 2006 年建立的 ${ }^{[1]}$. 他们利用逆转录病毒载体在小鼠成纤维 细胞中导入了 4 个与多能性有关的基因一Oct4, Sox2, $c-m y c$ 和 Klf4. 接着, 利用另一个多能性标志分子 Fbx15 的表达对转染后的细胞进行了篮选, 最终得到 了具有胚胎干细胞某些特性的多能性干细胞, 并命 名为“诱导产生的多能性干细胞”. 虽然他们得到的 细胞与真正的 $\mathrm{ES}$ 细胞相比还存在着许多差异, 但是 这一研究开创了利用少数几个因子来诱导多能性产 生的先例. 一年后, 3 家实验室各自的研究证实了该 结果的可靠性, 并且提高了产生的iPS细胞的质量 [16 18]. 他们用Nanog或者Oct4 的内源性表达作为篮选 标志取代了最初的Fbx15, 得到的iPS细胞在形态学、 基因表达状况和表观遗传修饰方面都与ES细胞十分 相似. 此外, 这些iPS细胞可以产生嵌合小鼠, 并能
够嵌合到生殖系中. 不久, 另外两项研究相继报道了 利用形态学作为篮选标准来建立iPS细胞的方法, 这 一方法在建立人类iPS细胞的研究中具有潜在的优势 [19,20]. 随后人类的iPS细胞系也建立起来了, 利用的 正是这样一种形态学的选择方法. 在新近的研究中, Yamanaka等人 ${ }^{[21]}$ 用他们在小鼠中使用过的 4 种因子 实现了人类皮肤成纤维细胞的重编程; 而Thomson实 验室 ${ }^{[22]}$ 选用的是他们自己篮选出的 4 种因子 Oct4, Sox2, Nanog和Lin28, 其中Oct4 与Sox2 在Yamanaka 的研究中也很关键. 紧随其后, Yamanaka小组又在原 有工作基础上取得了重要突破：从 4 个转录因子中去 掉了肿瘤相关因子 c-Myc, 使iPS细胞的生产更为安 全 ${ }^{[23]}$. 日前, 已有研究将iPS细胞用于治疗镰刀型贫 血症小鼠 24$]$. 在短短一年多的时间里, iPS细胞的建 立方法有了明显的改善, 并且成功地应用于人类细 胞的研究和疾病模型动物的治疗, 说明这一方法具 有可操作性和稳定性, 并且其安全性也在不断提高, 因此这种方法对将来的医学研究有着巨大的价值.

\section{3 iPS 细胞系建立的一些重要环节}

iPS 细胞建立的大致过程如下: 首先, 将几个重 要的多能性相关基因通过逆转录病毒转染的方法导 入小鼠或人类的成纤维细胞中; 其次, 基因导入一段 时间后, 通过药物或形态学特征对转染的细胞进行 选择; 最后, 篮选出的细胞要经过一系列严格的检验, 并与 ES 细胞进行比较, 从而才能证明其多能性.

图 1 显示了 iPS 细胞建立的简单过程. 一些重要 的环节将在下文中做详细的讨论.

\section{1 这几种因子的发现过程}

细胞的多能性受到许多因子精密而又复杂的调 控 ${ }^{[25]}$. ES细胞和体细胞融合后能诱导体细胞核的重 编程, 这一现象提示在 ES细胞中存在着一些因子, 这些因子对于多能性的建立可能至关重要. 虽然, 人 们对于这些与多能性建立有关的因子了解甚少, 但 对于与多能性维持相关的因子已经有了一定程度的 了解. 由于ES细胞自身具有重编程的活性, 因而这 些与多能性维持相关的因子很可能在多能性的建立 过程中也发挥着关键的作用 ${ }^{[26]}$. 基于这一假设, Takahashi和Yamanaka ${ }^{[1]}$ 对 24 个与多能性维持相关的候 选基因进行了研究, 检测它们能否诱导成体细胞产 生多能性. 这 24 个因子包括: ES细胞特异的转录因 


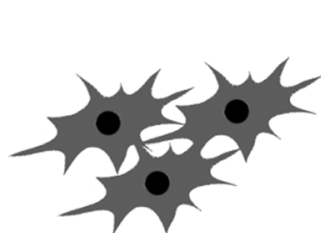

小鼠或人的成纤维细胞

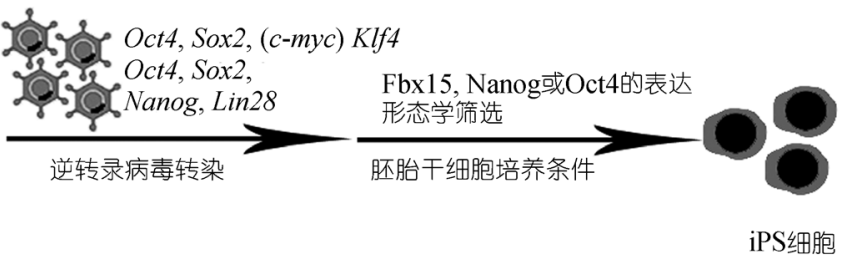

图 1 iPS 细胞的建立步骤
子, 如Oct4, Sox 2 和Nanog, 它们对于ES细胞和早期 胚胎的多能性维持有着必不可少的作用; 一些对 ES 细胞的增殖发挥重要作用的(原)癌基因产物, 如 Tcl1, Stat3, c-Myc和Eras; 其他对ES细胞起重要作用或是 在ES细胞中特异表达的分子, 如ECAT1, Esg1, Fbx15 和KIf4. 将所有的 24 个基因导入小鼠成纤维细胞之 后, 得到了表达Fbx15(细胞多能性的一个标志分子) 的细胞集落; 而任何单个基因的导入都无法产生这 样的集落. 他们继而尝试了从 24 个基因中依次去掉 其中的一个(即导入 23 个基因), 随后发现了 10 个基 因，其中任何一个被撤掉都会严重影响集落的产生; 而这 10 个基因的共同导入比 24 个基因的共同导入产 生了更多的集落. 他们接下来又从这 10 个基因中依 次去掉其中的任何一个(即导入 9 个基因), 进一步发 现了 4 个至关重要的基因, 其中任何一个的缺失都导 致集落形成受阻. 这 4 种基因的共同导入产生集落的 能力与先前那 10 种基因共同导入时的能力相似. 在 当时的条件下, 他们检测了这 4 种基因的不同组合方 式能否产生iPS细胞, 结果表明, 这 4 个基因中的任 意 2 个或 3 个组合, 都无法形成具有 ES细胞特性的 iPS细胞, 说明这 4 个基因缺一不可. 这就是这 4 个关 键基因(Oct4,Sox2, c-myc和 Klf4) 的发现过程. 此后, 除了 Thomson等人 ${ }^{[22]}$ 利用自己的方法篮选出了另外 一套基因组合之外，几乎其他所有关于iPS细胞的研 究都采用了Yamanaka和Takahashi所采用的这 4 种基 因.

Thomson等人的研究同样也得益于“ES细胞-体 细胞融合实验”的启发. 他们前期的工作发现, 人类 ES 细胞能够通过细胞融合实验使髓前体细胞 (myeloid precursor cells)发生重编程[9]. 最初, 他们比 较了人类ES细胞和髓前体细胞的基因表达谱, 挑选 出许多在 ES细胞中相对高表达的基因. 他们再根据 这些基因在多能性调控中的已知功能对其进行了排 序, 从中选出 14 个候选基因. 将这些候选基因以不 同的组合方式导入一种由人类ES细胞分化得到的间
充质样细胞后, 他们检验了不同基因的导入对这种 细胞重编程的作用. 最后, 他们锁定了 4 种因子Oct4, Sox2(这两种因子在Yamanaka等人的工作中也 被选用), Nanog以及Lin28. 随后, 他们用多种方法证 明了这 4 种因子的导入的确具有使人类新生儿成纤 维细胞发生重编程的能力.

最近, Yamanaka等人在前期工作的基础上对实 验过程进行了一定修改(提高了转染效率, 推迟了篮 选的时间), 得到了新的结果, 修正了最初的结论. 他 们发现, 从 4 个转录因子中去除c-Myc后也可以产生 iPS细胞, 但是, iPS细胞产生的效率比包含 $\mathrm{c}-\mathrm{Myc}$ 的 时候要低很多 ${ }^{[23]}$. 他们先后尝试了不同的供体细胞 (小鼠胚胎成纤维细胞(MEFs)、成年小鼠尾尖成纤维 细胞以及成年人成纤维细胞), 在这几种细胞中仅导 $\lambda$ Oct4, Sox 2 和Klf4 均可以获得iPS细胞. 他们尝试了 不同的篮选策略, 发现无论是用 Nanog 表达还是用 $F b x 15$ 表达进行药物筷选, 或者仅依赖形态学标准进 行篮选, 这 3 种因子都可以诱导 $\mathrm{PPS}$ 细胞的产生. 虽 然, 从 4 种因子中去除c-Myc会严重影响诱导效率, 但 $\mathrm{c}-\mathrm{Myc}$ 的去除有时候却能产生更为特异、质量更高 的iPS细胞 ${ }^{[23]}$. 更为重要的是, 从安全性的角度考虑, 因为 $c-M y c$ 有潜在的致癌作用(带有 $c-m y c$ 转基因的小 鼠有些产生了肿瘤, 这可能是 $c-m y c$ 基因的活化造成 的 ${ }^{[211}$ ), 它的去除对于iPS细胞研究应用于临床是一个 长足的进步.

\section{2 iPS 细胞的篮选}

在诱导的过程中, 监测细胞的变化并利用直观 的方法挑选出可能具有了多能性的细胞对整个工作 至关重要. Yamanaka小组在最初的研究中选用了 $F b x 15$ 作为篮选iPS细胞的报告基因. 尽管Fbx15 对于 多能性的维持和胚胎的发育来说并不是必不可少的, 但它在小鼠 ES 细胞和早期胚胎中有着非常特异的表 达. Yamanaka等人 “利用同源重组技术将 $\beta$-geo序列 (融合的 $\beta$-半乳糖苷酶基因和新霉素抗性基因)插入到 小鼠Fbx15 序列中, 希望当Fbx15 被激活时(即便只是 
部分激活), 细胞能产生抗性从而对普通剂量的 G418 耐受. 多能性相关基因导入 $3 \mathrm{~d}$ 后, G418 被添加至 细胞培养基中, 发生了转变的细胞(表达了 Fbx15 的 细胞)由于具有 G418 抗性而存活下来. 利用这种篮选 策略得到的细胞集落具有小鼠ES细胞的部分特征. 然而，这种细胞在基因表达模式和DNA甲基化模式 上都与ES细胞有所不同, 而且这种细胞无法产生发 育到期的嵌合小鼠. 由此可知, 这些细胞的重编程是 不完全的，而造成这一结果的原因很可能是选用的 筛选策略不够理想.

针对这一问题, Yamanaka小组 ${ }^{[16]}$ 以及Maherali ${ }^{[17]}$ 和Wernig ${ }^{[18]}$ 等人提出假设: 如果选用对多能性更为 关键的基因作为报告基因，得到的iPS细胞或许会具 备更多ES细胞的特性. 他们利用基因重组技术建立 了具有药物抗性基因的小鼠成纤维细胞, 这些细胞 的抗性受内源性Nanog或Oct4 表达的调控. 这两个转 录因子在细胞多能性维持方面的作用已被研究得相 当充分. 这 3 个实验室利用新的篮选策略均建立了稳 定的iPS细胞系，这些细胞系在各个方面都表现出与 ES细胞极为相似的特性. Wernig等人 ${ }^{[18]}$ 比较了 Nanog 和Oct4 的篮选效果, 发现利用Oct4 选出的抗性细胞 比利用Nanog选出的细胞在数量上少很多, 但前者中 获得 ES细胞特性的细胞比后者多. 这一结果提示, 在作为评判多能性的标准方面, Oct4 的激活比Nanog 的激活更为严格.

对于人类细胞来说，导入报告基因在技术上比 较困难, 而且可能会造成插入突变 ${ }^{[17]}$. 因此, 用形态 学的标准来代替报告基因的篮选是十分有价值的. Maherali等人 ${ }^{[17]}$ 发现，仅依靠形态学篮选可能就足以 建立iPS细胞系. 后来的两项研究证实了在不对供体 细胞做任何额外遗传修饰的情况下，仅利用形态学 的标准对细胞进行篮选也能够分离出小鼠的iPS细胞 [19,20]. 不久后, 利用这一形态学篮选策略, Yamanaka 实验室 ${ }^{[21]}$ 和 Thomson实验室 ${ }^{[22]}$ 均从人类体细胞中成 功地诱导出了iPS细胞.

除了篮选的方式以外，进行筛选的时间对于iPS 细胞的产生也有明显的影响. 在最初的研究中, Yamanaka小组 ${ }^{[1]}$ 在导入基因 $3 \mathrm{~d}$ 之后就开始用 G4 18 对表 达Fbx15 的细胞进行篮选. 后来的许多研究尝试在不 同的时间对特定基因的表达进行篮选. 随着篮选时 间的推迟，得到的抗性细胞集落在数量上是增加的. 造成这一现象的原因是, 细胞发生重编程而产生多
能性是一个渐变的过程, 多能性基因的激活也是随 着时间推移而逐渐发生的: 小鼠成纤维细胞被转染 后的 2 4 周内, 碱性磷酸酶、SSEA1 和Nanog的表达 相继开启, 暗示着重编程的逐渐完成 ${ }^{[18]}$.

\section{3 获得细胞的鉴定}

细胞的多能性要满足两个基本条件: 强大的自 我更新能力和分化潜能. 目前有很多种具体的指标 可用来评判获得的iPS细胞是否像真正的ES细胞一样 具有多能性, 如形态学标准、标志分子的表达、生长 特性、发育潜能、表观遗传学特征等. 图 2 总结了鉴 定iPS细胞的几个方法. 目前所建立的iPS细胞都可以 做到在形态和生长特性方面与 ES细胞一致, 其基因 表达谱与ES细胞的基因表达谱也基本上类似，只有 少部分基因的表达不同. iPS细胞也具有发育为 3 个 胚层细胞的能力, 并能参与生殖系的发育, 这与正常 的ES细胞相同. 大多数研究都鉴定了iPS细胞的表观 遗传学特征, 发现iPS细胞与ES细胞具有相似的DNA 甲基化模式和组蛋白修饰情况; Wernig等人 ${ }^{[18]}$ 建立的 iPS细胞与ES细胞一样，对整个基因组的去甲基化具 有耐受的能力; Maherali等人 ${ }^{[17]}$ 发现, 在iPS细胞发生 分化的同时，一条X染色体也发生了随机的失活. 更 为有趣的是, iPS细胞与MEFs 融合之后, 能够赋予 MEFs 类似于ES细胞的表型 ${ }^{[17]}$, 这是对iPS细胞多能 性的另一个严格检验.

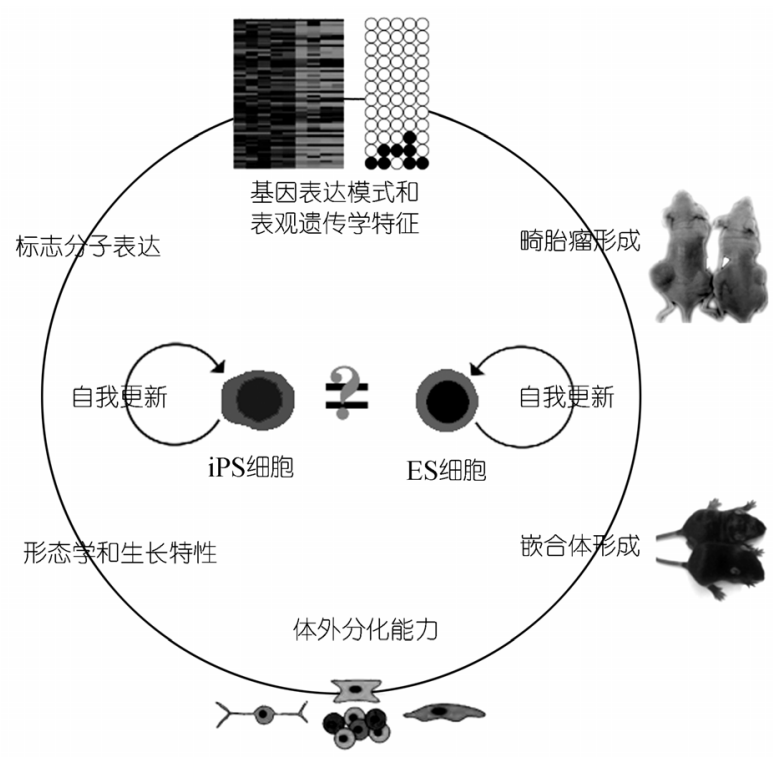

图 2 iPS 细胞的鉴定 


\section{4 相关的科学问题}

iPS 细胞的出现在为干细胞研究领域带来新理念 和新技术的同时，也产生了许多新的科学问题，等待 人们进一步去探索. 接下来我们将着重讨论这些相 关的重要科学问题.

4.1 仅仅几个因子的导入如何能够诱导体细胞发生 重编程而具有多能性?

根据目前的研究研究结果可知, ES 细胞的多能性 受到转录因子网络和表观遗传学的复杂调控, 因此仅 靠操纵几个基因的表达而诱导出多能性这一结果最初 是出乎意料的. 那么, 导入的这几个因子是如何完成这 一复杂任务的呢? 目前尚无直接的研究来回答这个问 题, 但是, 我们可以根据以往的研究来进行一些推测.

简单介绍一下这些研究中所用到的多能性相关因 子—OCt4/Sox $2 / \mathrm{Klf} 4 / \mathrm{c}-\mathrm{Myc}$ 和Oct $4 / \mathrm{Sox} 2 / \mathrm{Nanog} / \mathrm{Lin} 28$ 一在多能性调控中的作用. Oct 4 , Sox 2 和Nanog这 3 个转录因子在ES细胞多能性的维持中发挥的关键作 用已经为人们所熟知. Oct4 是多能性调控中一个至关 重要的因子, 缺失了 Oct4, 根本无法得到ES细胞; 要 维持多能性, Oct4 表达水平也必须受到严格控制, Oct4 表达不足或过多都会导致ES细胞的分化 ${ }^{[27,28]}$. Oct4 对于很多基因的调控需要与Sox 2 进行协同作用 [29]. Sox2 的表达虽然不局限于多能性细胞 ${ }^{[30]}$, 但它对 早期胚胎发育和抑制分化也十分重要. 缺乏Sox2 的 胚胎无法形成上胚层(epiblast) ${ }^{[30]}$, 在ES细胞中阻断 Sox2 的功能造成ES细胞的分化 ${ }^{[31]}$. Nonog的转录受 Oct4 和Sox 2 的共同调节 ${ }^{[32]}$ ，它的缺乏将导致细胞自 发地向原始内胚层细胞分化 ${ }^{[33,34]}$. 基因组范围的分 析结果发现，在小鼠和人类的ES细胞中, Oct4, Sox2 和Nanog共同调控着许多靶基因, 其中很多基因的产 物是对发育至关重要的转录因子 ${ }^{[35,36]}$. Oct4, Sox2 和 Nanog三个转录因子一起形成了ES细胞的调控系统, 其中牵涉了自调节和前馈等多种机制. $c-m y c$ 是一种 广为人知的原癌基因, 它对于某些成体干细胞的自我 更新起一定作用 ${ }^{[37,38]} \cdot c-m y c$ 是Lif/STAT3 和Wnt信号 通路的一个主要的下游基因 ${ }^{[39,40]}$, 这两条信号通路 对于多能性的维持都很重要. Klf4 具有癌基因和肿瘤 抑制基因的双重特性 2 . 它的过表达可以维持Oct4 的表达, 并抑制ES细胞的分化 ${ }^{[41]}$; 它可以协同Oct4 和Sox 2, 从而调节某些基因的转录 ${ }^{[42]}$. Lin 28 在许多 物种当中是一种控制多种细胞发育的负调控基因 ${ }^{[43]}$.
在人类和小鼠 ES细胞中, 随着 ES细胞的分化, $\operatorname{Lin} 28$ 的表达下降 ${ }^{[44]}$.

上述转录因子与靶基因的调节作用与靶基因的 可及性有关, 而这种可及性受到DNA、组蛋白和染色 质结构修饰的影响 ${ }^{[45]}$. ES细胞的基因组有着很高的 可塑性, 其染色质结构比较开放, 具有转录活性; 而 体细胞的基因组可塑性比较差. c-Myc可以诱导体细 胞染色质结构发生改变 ${ }^{[40]}$. 也许, 导入成纤维细胞的 c-Myc首先发挥了这一作用, 使Oct4, Sox2 和Klf4 等 因子得以对靶基因的转录进行调节, 从而改变细胞 的命运 ${ }^{[26]}$. 后来的研究结果表明, 对实验策略进行稍 作修改之后, 只导入 Oct4, Sox2 和Klf4 也能够实现成 纤维细胞的重编程而产生iPS细胞 ${ }^{[23]}$. 在这种情况下, Oct4, Sox2 和Klf4 可能通过募集内源性的c-Myc蛋白 而诱导了重编程的发生. 由于成纤维细胞中内源性 $\mathrm{c}-\mathrm{Myc}$ 蛋白的表达水平比较低, 所以与外源性 c-Myc 存在时相比, 不导入c-Myc时重编程的速度减慢了, 效率也降低了.

另一方面, Thomson等人 ${ }^{[22]}$ 的研究表明, Oct4, Sox 2, Nanog和Lin 28 这 4 个基因的组合导入也可以诱 导人类成纤维细胞转变为iPS细胞. 这证明iPS细胞的 产生可以通过不同的因子组合来诱导. 这 4 个因子是 否与Yamanaka小组的 4 个因子有着相似的作用方式, 现在还不得而知. 目前, 人们对于因子诱导的重编程 现象背后所蕴藏的机理还没有进行直接的研究; 今 后, 对iPS细胞产生的分子机理有了透彻的了解之后, 每个因子在这个过程中所扮演的角色才能够逐一地 被解读.

\section{2 iPS 细胞为何能够实现正常的分化?}

由于多能性相关因子的导入采用了持续表达的 逆转录病毒载体, 如果这些外源基因持续地表达, iPS 细胞为什么能正常分化呢? 这些外源基因的持续 表达对于多能性的维持是否是必要的呢?

实际上, 在iPS细胞中, 导入的这些基因的总体表 达水平 (内源表达和外源表达)与ES细胞中几乎是一致 的. 进一步研究揭示, 这些基因的表达大部分来源于 内源基因的激活, 而导入的外源基因表达水平很低. Wernig等人 ${ }^{[18]}$ 研究发现, 在MEFs向iPS细胞转变的过 程中, 外源基因的表达逐渐发生了沉默, 这一过程伴 随着多能性标志分子的相继出现, 暗示iPS细胞中存在 特定的机制调控这些多能性相关基因的表达量. 启动 子DNA的甲基化会抑制基因的转录活性, 而DNA的甲 
基化是由DNA甲基转移酶来实现的. 研究发现, 导入 的外源基因的表达水平与Dnmt3a2 (一种控制DNA发 生从头甲基化的甲基转移酶)的表达水平成反比 ${ }^{[16]}$, 因此, 导入的外源基因在重编程过程中逐渐失活的原 因很可能是这些基因发生了从头甲基化. 由此可见, iPS细胞自身具备调节多能性相关基因表达的机制, 因而, iPS细胞多能性的维持并不需要外源基因的持续 表达. 同时, 外源基因的适时沉默也确保了iPS细胞能 够像ES细胞那样正常地发生分化.

\section{3 iPS 细胞的产生效率为何较低?}

目前, 研究人员获得iPS细胞的效率依然较低, 大约在万分之几到千分之几之间. 其主要原因很可 能与逆转录病毒转染这种基因导入手段的缺陷有关. 逆转录病毒的转染是一种相对随机且不易控制的过 程. 目的基因的整合位点、插入的拷贝数等都无法人 为掌控. 很可能导入的每种因子的表达水平都必须 合适才能让细胞发生重编程, 而这种合适的水平可 能范围又很窄 ${ }^{[1]}$ : 正如前面所提到的, Oct4 表达量的 升高或降低都会导致ES细胞的分化 ${ }^{[27]}$. 因此, 这种 随机的基因导入方式导致只有很少的细胞才具备发 生重编程的条件. 另外, 有人怀疑 IPS细胞的获得效 率低与iPS细胞的来源有关: iPS细胞的来源可能存在 于成纤维细胞中极少数的皮肤干细胞. 然而, 用富集 了多种成体干细胞的骨髓细胞建立iPS细胞之后发现, 利用骨髓细胞作为供体细胞, 并不能提高iPS细胞的 获得效率 ${ }^{[1]}$. 直接利用皮肤干细胞来进行iPS细胞研 究可能会更直接地验证这一可能性. 此外, 还有几种 可能性值得考虑: 转基因的整合位点必须特异才能 引起重编程, 重编程还需要其他未知基因的导入或 者需要细胞发生一些未知的遗传学或表观遗传学的 改变 ${ }^{[21]}$ 等. 目前, 人们尚无法确切地解释这个问题. 这一问题的解答和解决在很大程度上依赖于将来对 iPS细胞产生机理的揭示.

然而, 从实际的角度来考虑, 即便 iPS 细胞产生 的效率不高, 但只要能得到稳定的 iPS 细胞系, 对于 很多研究来说已经足够了. 与 ES 细胞的建立和核移 植等方法相比, iPS 细胞的获得效率相对较高, 这也 是这个方法的一大优势所在.

\section{$5 \mathrm{iPS}$ 细胞研究的应用价值和发展方向}

\section{1 应用价值}

iPS细胞的出现, 在干细胞研究领域、表观遗传
学研究领域以及生物医学研究领域都引起了强烈的 反响, 这不仅是因为它在基础研究方面的重要性, 更 是因为它为人们带来的光明的应用前景. 在基础研 究方面, iPS细胞为研究细胞核重编程提供了一个全 新的视角. 它的出现, 已经让人们对多能性的调控机 制有了突破性的新认识; 利用iPS细胞作为实验模型, 只操纵几个因子的表达, 这更会大大加速对多能性 调控机理的深入研究. 在实际应用方面, iPS细胞的 获得方法相对简单和稳定, 不需要使用卵母细胞或 者胚胎, 这在技术上和伦理上都比其他方法更有优 势. 利用这一技术人们可以制造特定病人来源的iPS 细胞, 可望用“个性化”的移植来治疗诸多疾病. 目前, 将iPS细胞应用于疾病治疗的研究在小鼠模型上取得 了重要的进展: Hanna等人 ${ }^{[24]}$ 用镰刀型贫血症小鼠的 皮肤成纤维细胞建立了iPS细胞, 通过对iPS细胞的改 造和分化得到了具有正常功能的造血前体细胞, 从 而治疗了镰刀型贫血症的小鼠. 这对于iPS细胞用于 人类疾病的治疗起到了强有力的推动作用, 更带来 了希望和信心. 同时, 人们也可以制造出特定疾病来 源的iPS细胞, 用于疾病发生机理的研究和新药的开 发.

\section{2 发展方向}

iPS 细胞研究在今后的发展中会兼顾对基础科学 问题的解答和对实际应用价值的开发这两个方面.

首先, 人们一定会不遗余力地去探究控制 iPS 细 胞产生的具体机制, 进一步加深对重编程和多能性 调节机理的认识. 导入的几种因子各自有着怎样的 贡献? 它们起作用的时间顺序是怎样的? 它们的表 达水平需要在怎样的范围内才能有作用? 有哪些因 子是可以被其他因子替代的? 这些都是人们迫切想 知道答案的问题, 也是今后的一个研究重点. 由于目 前采用的这种病毒导入基因的方法在很大程度上是 随机和难以控制的, 想要用这种方法来精确地研究 因子的剂量对于重编程的作用是比较困难的. 在以 后的研究中, 可以利用同源重组的技术直接对多能 性相关基因的启动子进行改造, 使这些基因的表达 时间和表达量可以人为地加以控制, 从而更好地研 究每个基因对于重编程和多能性的调控作用. 此外, 还可以通过人为调控这些基因的开启顺序来更深入 地研究因子诱导重编程发生的过程和机理. 另一方 面, 目前的研究结果已经提示几种不同的因子组合 都能够起到诱导重编程的作用, 那么, 在实验条件不 
断完善的情况下，人们可以继续尝试篮选其他候选 基因，寻找更高效、更安全的因子组合.

其次, 基于目前对成体干细胞的研究热情和进 展, 人们会考虑将iPS细胞研究与成体干细胞研究相 结合. 由于成体干细胞具有一定的分化潜能, 其基因 组具备一定可塑性, 某些成体干细胞自身也表达一 些多能性相关分子, 人们曾经推测, 成体干细胞可能 更容易发生重编程. 然而, 在核移植实验中, 人们比 较过成体干细胞和分化细胞的重编程效果, 发现分 化的细胞重编程的情况却比成体干细胞更理想 ${ }^{[46,47]}$. 今后, 利用 $i P S$ 细胞的建立, 人们可以更直观地研究 成体干细胞参与重编程的过程, 也可以从这个角度 更深入地了解重编程的机制. 不久前已经有研究报 道, Oct4 的导入使小鼠毛囊间基底层表皮细胞(其中 富集了表皮干细胞)发生了去分化 ${ }^{[48]}$. 相信今后这方 面的工作会取得更多重要的进展.

再次, 虽然利用 iPS 细胞来制备用于疾病治疗的 多能性干细胞在理论上是可行的, 但完美解决 iPS 细胞的安全性问题尚需时日. 在今后的研究中应尽 量避免肿瘤相关基因(如 $c-m y c$ )的使用, 取而代之的 是那些没有副作用的重编程因子. 同时, 逆转录病毒 转染可能会导致一些癌基因的激活, 也可能导致某 些重要基因功能受阻, 今后应尝试其他的诱导方式, 如腺病毒转染、可穿透细胞的重组蛋白质等. 还有一 种办法能解决上述两个问题，即寻找到可以用于替 代这些多能性相关基因的小分子药物, 这需要今后 对调控重编程的信号通路进行大量深入的研究.
最后, iPS 细胞对医学研究最直接的益处在于对 疾病机理研究和新药开发等方面的贡献. 通过对 ES 细胞进行遗传改造, 人们可以在体外重建某些疾病 的模型; 而直接利用来自病人的 iPS 细胞, 重建疾病 模型的过程会得到简化, 模型仿真性也会提高. 世界 上有很多家族遗传的疾病, 充分利用这些疾病资源, 建立疾病特异的 iPS 细胞库, 利用这种简化的模型, 人们更容易揭示这些疾病的发病机制, 从而有望从 根本上寻找到治疗的途径. 另一方面, iPS 细胞为新 药的开发提供了理想的模型: 药物的毒性和有效性 甚至作用机理都可以在 iPS 细胞或由 iPS 细胞产生的 特定细胞中得到研究. 因此, 如何实现 iPS 细胞在体 外定向分化为能模拟体内情况的功能性细胞也将成 为今后研究的重点.

\section{6 结束语}

从 iPS 细胞首次在实验动物中诞生, 到 iPS 细胞 建立方法的逐渐完善, 再到 iPS 细胞研究在人类细胞 中获得成功, 最后到 iPS 细胞应用于疾病模型动物的 治疗, 整个过程只用了短短一年半的时间. 这种快速 发展的原因在于对过去大量知识的积累和运用, 更 在于观念上的大胆创新. 今后, iPS 细胞的研究会成 为生命科学领域的一个重要方向, 随着技术的不断 进步, 相信 iPS 细胞研究的进展会比人们想象中更为 迅速. 今天, 我们从 iPS 细胞上看到了它为人类疾病 治疗所带来的希望和契机, 我们更期待在不远的将 来, iPS 细胞的不足会在人们的努力下逐一地被克服, iPS 细胞最终有望真正用于造福人类健康.

\section{参考文献}

1 Takahashi K, Yamanaka S. Induction of pluripotent stem cells from mouse embryonic and adult fibroblast cultures by defined factors. Cell, 2006, 126(4): 663-676[DOI]

2 Spivakov M, Fisher A G. Epigenetic signatures of stem-cell identity. Nat Rev Genet, 2007, 8(4): 263-271 [DOI]

3 Hochedlinger K, Jaenisch R. Nuclear reprogramming and pluripotency. Nature, 2006, 441(7097): 1061 - 1067 [DOD]

4 Fujimori T, Kurotaki Y, Miyazaki J, et al. Analysis of cell lineage in two- and four-cell mouse embryos. Development, 2003, 130(21): $5113-5122$ [DOI]

5 Rodolfa K T, Eggan K. A transcriptional logic for nuclear reprogramming. Cell, 2006, 126(4): 652 - 655[DOI]

6 Tada M, Takahama Y, Abe K, et al. Nuclear reprogramming of somatic cells by in vitro hybridization with ES cells. Curr Biol, 2001, 11(19): $1553-1558 \underline{[\mathrm{DOI}]}$

7 Tada M, Morizane A, Kimura H, et al. Pluripotency of reprogrammed somatic genomes in embryonic stem hybrid cells. Dev Dyn, 
2003, 227(4): 504-510[DOI] ence, 2005, 309(5739): 1369-1373[DOI]

10 Hansis C, Barreto G, Maltry N, et al. Nuclear reprogramming of human somatic cells by xenopus egg extract requires Brg1. Curr Biol, 2004, 14(16): 1475-1480[DOI]

11 Taranger C K, Noer A, Sorensen A L, et al. Induction of dedifferentiation, genomewide transcriptional programming, and epigenetic reprogramming by extracts of carcinoma and embryonic stem cells. Mol Biol Cell, 2005, 16(12): 5719—5735[DOI]

Matsui Y, Zsebo K, Hogan B L. Derivation of pluripotential embryonic stem cells from murine primordial germ cells in culture. Cell, 1992, 70(5): 841-847 $\underline{\text { DOI] }}$

13 Jiang Y, Jahagirdar B N, Reinhardt R L, et al. Pluripotency of mesenchymal stem cells derived from adult marrow. Nature, 2002, 418(6893): 41-49 $\underline{\text { [DOI] }}$

14 Kanatsu-Shinohara M, Inoue K, Lee J, et al. Generation of pluripotent stem cells from neonatal mouse testis. Cell, 2004, 119(7): 10011012[DOI]

15 Guan K, Nayernia K, Maier L S, et al. Pluripotency of spermatogonial stem cells from adult mouse testis. Nature, 2006, 440(7088): 1199-1203[DOI]

Okita K, Ichisaka T, Yamanaka S. Generation of germline-competent induced pluripotent stem cells. Nature, 2007, 448(7151): 313$317 \underline{\text { DOI] }}$

17 Maherali N, Sridharan R, Xie W, et al. Directly reprogrammed fibroblasts show global epigenetic remodeling and widespread tissue contribution. Cell Stem Cell, 2007, 1(1): 55-70[DOI]

Wernig M, Meissner A, Foreman R, et al. In vitro reprogramming of fibroblasts into a pluripotent ES-cell-like state. Nature, 2007, 448(7151): 318-324[DOI]

19 Meissner A, Wernig M, Jaenisch R. Direct reprogramming of genetically unmodified fibroblasts into pluripotent stem cells. Nat Biotechnol, 2007, 25(10): 1177-1181 [DOI]

20 Qin D, Li W, Zhang J, et al. Direct generation of ES-like cells from unmodified mouse embryonic fibroblasts by Oct4/Sox2/Myc/Klf4. Cell Res, 2007, 17(11): 959-962[DOI]

21 Takahashi K, Tanabe K, Ohnuki M, et al. Induction of pluripotent stem cells from adult human fibroblasts by defined factors. Cell, 2007, 131(5): 861-872[DOI]

22 Yu J, Vodyanik M A, Smuga-Otto K, et al. Induced pluripotent stem cell lines derived from human somatic cells. Science, 2007, 318(5858): 1917-1920[DOI]

Takahashi K, Okita K, Nakagawa M, et al. Generation of high quality iPS cells. Neurosci Res, 2007, 58(Suppl1): S19 [DOI]

Hanna J, Wernig M, Markoulaki S, et al. Treatment of sickle cell anemia mouse model with iPS cells generated from autologous skin. Science, 2007, 318(5858): 1920-1923 $\underline{\text { [DOI] }}$

Yamanaka S, Li J, Kania G, et al. Pluripotency of embryonic stem cells. Cell Tissue Res, 2007, 2008, 331(1): 5-22

Yamanaka S. Strategies and new developments in the generation of patient-specific pluripotent stem cells. Cell Stem Cell, 2007, 1(1): $39-49[\mathrm{DOI}]$

Niwa H, Miyazaki J, Smith A G. Quantitative expression of Oct-3/4 defines differentiation, dedifferentiation or self-renewal of ES cells. Nat Genet, 2000, 24(4): 372-376[DOI]

Nichols J, Zevnik B, Anastassiadis K, et al. Formation of pluripotent stem cells in the mammalian embryo depends on the POU transcription factor Oct4. Cell, 1998, 95(3): 379-391ㅁO]

Masui S, Nakatake Y, Toyooka Y, et al. Pluripotency governed by Sox2 via regulation of Oct3/4 expression in mouse embryonic stem cells. Nat Cell Biol, 2007, 9(6): 625-635[DOI]

30 Avilion A A, Nicolis S K, Pevny L H, et al. Multipotent cell lineages in early mouse development depend on Sox2 function. Genes Dev, 2003, 17(1): 126-140[DOI]

31 Ivanova N, Dobrin R, Lu R, et al. Dissecting self-renewal in stem cells with RNA interference. Nature, 2006, 442(7102): 533$538 \underline{[\mathrm{DOI}]}$

Rodda D J, Chew J L, Lim L H, et al. Transcriptional regulation of Nanog by OCT4 and SOX2. J Biol Chem, 2005, 280(26): 24731- 
$24737 \underline{[\mathrm{DOI}]}$

Mitsui K, Tokuzawa Y, Itoh H, et al. The homeoprotein Nanog is required for maintenance of pluripotency in mouse epiblast and ES cells. Cell, 2003, 113(5): 631-642 [DOI]

Chambers I, Colby D, Robertson M, et al. Functional expression cloning of Nanog, a pluripotency sustaining factor in embryonic stem cells. Cell, 2003, 113(5): 643-655[DOI]

Boyer L A, Lee T I, Cole M F, et al. Core transcriptional regulatory circuitry in human embryonic stem cells. Cell, 2005, 122(6): 947$956 \underline{\mathrm{DO}]}$

36 Loh Y H, Wu Q, Chew J L, et al. The Oct4 and Nanog transcription network regulates pluripotency in mouse embryonic stem cells. Nat Genet, 2006, 38(4): 431-440[DOI]

37 Waikel R L, Kawachi Y, Waikel P A, et al. Deregulated expression of c-Myc depletes epidermal stem cells. Nat Genet, 2001, 28(2): 165$168 \underline{\text { DOI] }}$

38 Satoh Y, Matsumura I, Tanaka H, et al. Roles for c-Myc in self-renewal of hematopoietic stem cells. J Biol Chem, 2004, 279(24): $24986-24993 \underline{[\mathrm{DOI}]}$

39 Cartwright P, McLean C, Sheppard A, et al. LIF/STAT3 controls ES cell self-renewal and pluripotency by a Myc-dependent mechanism. Development, 2005, 132(5): 885-896[DOD]

40 Adhikary S, Eilers M. Transcriptional regulation and transformation by Myc proteins. Nat Rev Mol Cell Biol, 2005, 6(8): 635645 [DOI]

41 Li Y, McClintick J, Zhong L, et al. Murine embryonic stem cell differentiation is promoted by SOCS-3 and inhibited by the zinc finger transcription factor Klf4. Blood, 2005, 105(2): 635-637 $\underline{\text { [DOI] }}$

42 Nakatake Y, Fukui N, Iwamatsu Y, et al. Klf4 cooperates with Oct3/4 and Sox2 to activate the Lefty1 core promoter in embryonic stem cells. Mol Cell Biol, 2006, 26(20): 7772—7782 [DOI]

43 Moss E G, Tang L. Conservation of the heterochronic regulator Lin-28, its developmental expression and microRNA complementary sites. Developmental Biology, 2003, 258(2): 432-442 [DOI]

44 Richards M, Tan S P, Tan J H, et al. The transcriptome profile of human embryonic stem cells as defined by SAGE. Stem Cells, 2004, 22(1): $51-64 \underline{[\mathrm{DOI}]}$

45 Jaenisch R, Bird A. Epigenetic regulation of gene expression: How the genome integrates intrinsic and environmental signals. Nat Genet, 2003, 33 (Suppl): 245-254 $\underline{\text { [DOI] }}$

46 Inoue K, Ogonuki N, Miki H, et al. Inefficient reprogramming of the hematopoietic stem cell genome following nuclear transfer. J Cell Sci, 2006, 119(Pt 10): 1985-1991[DOI]

47 Sung L Y, Gao S, Shen H, et al. Differentiated cells are more efficient than adult stem cells for cloning by somatic cell nuclear transfer. Nat Genet, 2006, 38(11): 1323-1328 $\underline{\text { [DOI] }}$

48 Grinnell K L, Yang B, Eckert R L, et al. De-differentiation of mouse interfollicular keratinocytes by the embryonic transcription factor Oct-4. J Invest Dermatol, 2007, 127(2): 372-380 [DOI] 